\title{
LA EVALUACIÓN DEL DESEMPEÑO DOCENTE: ANÁLISIS DE SUS RESULTADOS
}

\author{
Mstr. Sebastián Granda Merchán *
}

$\int$

a política de evaluación de la Universidad Politécnica Salesiana contempla, entre otros aspectos, la evaluación periódica del desempeño de los docentes. Hasta el momento, en la Sede Quito se han puesto en ejecución tres evaluaciones del desempeño docente: la primera en el semestre marzo/2001 - septiembre/2001, la segunda en el semestre octubre/2001 - febrero de 2002 y la tercera en el semestre marzo/2002 - septiembre/2002.

A continuación, se presentan algunos resultados de dichas evaluaciones así como su interpretación aunque incipiente aún.

\section{LA EVALUACIÓN DEL DESEMPEÑO DOCENTE}

Un primer dato que arrojan las evaluaciones implantadas en los tres últimos semestres es que los directores, docentes y estudiantes de la UPS-Q han evaluado el desempeño de los docentes en parámetros altos. Fijémonos en los promedios de evaluación del desempeño docente':

\begin{tabular}{|l|c|}
\hline Modalidad & $\begin{array}{l}\text { Promedio de los tres semestres } \\
\text { (desde el punto de vista de los es- } \\
\text { tudiantes, los directores y los do- } \\
\text { centes) sobre 5 }\end{array}$ \\
\hline Modalidad Presencial & 4,40 \\
\hline Modalidad Semipresencial & 4,46 \\
\hline
\end{tabular}

Un segundo dato es que los directores, docentes y estudiantes han evaluado cada vez mejor el desempeño de los docentes a lo largo de estos tres últimos semestres. Revisemos los promedios de evaluación de cada uno de los semestres:

Responsable del Área de Evaluación de la Dirección Académica de la UPS Sede Quito.

Todos los datos sobre las evaluaciones del desempeño docente que aparecen en el presente artículo fueron proporcionados por el Departamento de Informática de la UPS Sede Quito. 
Modalidad Presencial:

\begin{tabular}{|l|c|c|c|c|}
\hline Semestre & Primero & Segundo & Tercero \\
\cline { 4 - 5 } Promedios sobre 5 & 4,38 & 4,33 & 4,50 \\
\hline Porcentajes (aprox.) & $87 \%$ & $87 \%$ & $90 \%$ \\
\hline
\end{tabular}

\section{Modalidad Semipresencial:}

\begin{tabular}{|c|c|c|c|}
\hline Semestre & Primero & Segundo & Tercero \\
\hline Promedios sobre 5 & 4,30 & 4,50 & 4,58 \\
\hline Porcentajes (aprox.) & $86 \%$ & $90 \%$ & $92 \%$ \\
\hline
\end{tabular}

En el caso de la Modalidad Presencial se evidencia un ligero decremento del promedio en el segundo semestre pero una recuperación importante en el tercer semestre: 3 puntos porcentuales del segundo al tercer semestre. En la Modalidad Semipresencial, en cambio, constatamos un incremento progresivo del promedio a lo largo de los tres últimos semestres: 6 puntos porcentuales del primero al tercer semestre.

La escala que se utiliza en la UPS-Q para evaluar el desempeño de los docentes es la siguiente:

$$
\begin{array}{lr}
\text { Totalmente de acuerdo }= & 5 / 5 \\
\text { De acuerdo }= & 4 / 5 \\
\text { Parcialmente de acuerdo }= & 3 / 5 \\
\text { En desacuerdo }= & 2 / 5 \\
\text { Totalmente en desacuerdo }= & 1 / 5
\end{array}
$$

Si se utiliza esta escala para valorar los datos arriba expuestos, se puede concluir que el desempeño docente ha satisfecho -con mucho- las expectativas de los directores de escuela, de los estudiantes y de los mismos docentes.

Ahora bien, ¿qué nos dicen los datos sobre el nivel de desempeño docente?, ¿el hecho de que los directores, docentes y estu- 
diantes hayan evaluado alto el desempeño de los docentes significa que el nivel de desempeño docente ha sido también alto? No necesariamente. La razón fundamental se ubica en el proceso mismo de evaluación y en la validez de sus resultados.

La validez de los resultados de un proceso de evaluación del desempeño docente, como de cualquier proceso de evaluación, depende de muchos factores. Depende, por ejemplo, de los indicadores que se utilizan para evaluar las diferentes dimensiones del desempeño docente, de los instrumentos usados para la recolección de la información, de los criterios con los cuales se evalúa, entre otros. Así, es imposible que un proceso de evaluación arroje resultados válidos del desempeño docente si los indicadores que se utilizan para evaluar son ambiguos y no expresan con claridad las dimensiones a ser evaluadas, si los instrumentos carecen de precisión, o si los criterios que se utilizan para evaluar no mantienen relación con el ámbito que se pretende evaluar.

Sin desconocer que en el proceso de evaluación del desempeño docente puesto en marcha en la UPS-Q haya existido ciertos problemas con los indicadores (algunos no expresan con claridad las dimensiones del desempeño docente que se pretende evaluar) y los instrumentos de evaluación (ciertas preguntas sufren de imprecisión), es necesario destacar que un problema relevante tiene que ver con los criterios a partir de los cuales se ha evaluado el desempeño de los docentes.

No se han investigado aún las percepciones que los directores, docentes y estudiantes tienen de la docencia universitaria ni los criterios con los que evalúan el desempeño docente, pero por conversaciones mantenidas con algunos de ellos, diera la impresión de que, muchas veces, se lo hace a partir de criterios ajenos a los principios de la docencia universitaria. Pensemos, por ejemplo, en algunos de los criterios con los que los estudiantes evalúan el desempeño de sus docentes: para algunos el nivel de exigencia de los docentes hace referencia a la cantidad de conocimientos que ellos exigen memorizar o a la cantidad de deberes que exigen realizar. El nivel de preparación científico-académico de los docentes hace alusión a la cantidad de información que estos poseen y están en condiciones de transmitir. 
Las constataciones anteriores nos fuerzan, por el momento, a leer los resultados de las evaluaciones más como expresiones de la percepción que los diferentes actores universitarios tienen del desempeño docente, que como expresiones del nivel de desempeño mismo. Solo cuando los indicadores de evaluación y los instrumentos de recolección de información estén suficientemente pulidos, y cuando los criterios de evaluación estén acordes con los principios de la docencia universitaria, los resultados de las evaluaciones podrán ser leídos como datos válidos de la situación del desempeño docente.

Ahora bien, lo que los resultados de las evaluaciones del desempeño docente sí evidencian, es que en la UPS-Q ha existido, en estos tres últimos semestres, un alto nivel de correspondencia entre los criterios a partir de los cuales los docentes ejercen la docencia y los criterios con los que los directores, los estudiantes y los mismos docentes evalúan dicho ejercicio. En efecto, es debido a que los estudiantes, los directores y los docentes han evaluado el desempeño docente con los criterios que se corresponden con el ejercido de la docencia, que los resultados de las evaluaciones son tan halagadores.

En ningún momento dicho nivel de correspondencia debe ser entendido como el resultado de una situación de complicidad consciente entre los directores, docentes y estudiantes. Lejos de aqueIlo, pensamos que el alto nivel de correspondencia debe ser asumido, más bien, como el producto de un acuerdo inconsciente en torno al significado de la docencia universitaria.

\section{DIFERENCIAS EN LA EVALUACIÓN DEL DESEMPEÑO DO- CENTE}

Los resultados de las evaluaciones evidencian que los directores de escuela y los docentes han evaluado el desempeño docente mejor que los estudiantes. Revisemos los promedios de evaluación de cada uno de los actores: 
Modalidad Presencial:

\begin{tabular}{|c|c|c|}
\hline $\begin{array}{c}\text { Actores que han } \\
\text { evaluado el } \\
\text { desempeño docente }\end{array}$ & $\begin{array}{c}\text { Promedios de } \\
\text { evaluación de los tres } \\
\text { semestres sobre } 5\end{array}$ & Porcentajes \\
\hline Estudiantes & 4,17 & $83 \%$ \\
\hline Directores & 4,51 & $90 \%$ \\
\hline Docentes (autoevaluación) & 4,53 & $91 \%$ \\
\hline
\end{tabular}

Modalidad Semipresencial:

\begin{tabular}{|l|c|c|}
\hline $\begin{array}{c}\text { Actores que han } \\
\text { evaluado el } \\
\text { desempeño docente }\end{array}$ & $\begin{array}{c}\text { Promedios de } \\
\text { evaluación de los tres } \\
\text { semestres sobre 5 }\end{array}$ & Porcentajes \\
\hline Estudiantes & 4,27 & $85 \%$ \\
\hline Directores & 4,62 & $92 \%$ \\
\hline Docentes (autoevaluación) & 4,48 & $90 \%$ \\
\hline
\end{tabular}

En la Modalidad Presencial la diferencia entre el promedio de los estudiantes y el de los directores es de 7 puntos porcentuales, mientras que la diferencia entre el promedio de los estudiantes y el de los docentes es de 8 puntos. En el caso de la Modalidad Presencial la diferencia es de 7 y 5 puntos porcentuales, respectivamente.

Si analizamos los datos ya no por modalidades sino por facultades, constatamos que la tendencia anterior se repite pero con una novedad interesante: mientras menor es el promedio de evaluación de los estudiantes mayor es el promedio de los directores y/o de auto evaluación. Las tres facultades con más bajos promedios en la evaluación de los estudiantes son aquellas que tienen los más altos promedios en la evaluación de los directores y/o en la autoevaluación.

¿A qué se debe la diferencia entre el promedio de evaluación de los estudiantes y el de los directores-docentes? 
Si se parte de la constatación de que los directores y los estudiantes evalúan diferentes aspectos del desempeño docente (mientras los directores evalúan fundamentalmente el desempeño de los docentes fuera del aula, los estudiantes evalúan el desempeño docente dentro del aula), se puede concluir que la diferencia entre el promedio de evaluación de los directores y el de los estudiantes se debe, posiblemente, a que los profesores están se desempeñan mejor fuera del aula que dentro de ella.

El problema surge cuando intentamos explicar la diferencia del promedio de evaluación de los estudiantes y el de los docentes, puesto que -en términos generales- ambos actores evalúan los mismos aspectos. ¿Cómo explicar este fenómeno?

Si se asume la idea de que todo proceso de evaluación del desempeño docente -con o sin fines punitivos-genera presión sobre los docentes, se puede plantear la hipótesis de que el alto promedio de autoevaluación obedece, en términos generales, a una franca necesidad de los docentes de legitimar su desempeño: los docentes evalúan alto su desempeño para compensar la posible evaluación baja por parte de los directores y sobre todo, de los estudiantes. No es una coincidencia que las tres facultades en las que los estudiantes han evaluado más bajo el desempeño de los docentes, sean las mismas en las que los docentes se han autoevaluado más alto.

\section{FORTALEZAS Y DEBILIDADES DEL DESEMPEÑO DOCEN- TE DESDE EL PUNTO DE VISTA DE LOS ESTUDIANTES}

Como vimos anteriormente, los estudiantes de la UPS-Q han evaluado alto el desempeño de los docentes en estos tres últimos semestres: 4,17/5 en la Modalidad Presencial y 4,27/5 en la Modalidad Semipresencial. Si analizamos la forma como los estudiantes han evaluado cada una de las tres dimensiones del desempeño docente (sus actitudes, competencias metodológicas y competencias científico-académicas) encontramos algunas cuestiones interesantes: la dimensión del desempeño docente mejor evaluada por los estudiantes es la actitudinal, luego la científico-académica y por último, la que tiene que ver con las competencias metodológicas. 
Revisemos algunos datos:

Tanto en la Modalidad Presencial como Semipresencial la mayor parte de preguntas que se refieren a la dimensión actitudinal del desempeño docente se han ubicado en los primeros lugares y en posiciones altas. En este sentido, es interesante verificar como las preguntas \# 13 (El docente/tutor se relaciona con los estudiantes en forma respetuosa), \# 16 (Demuestra estar comprometido con su materia y la docencia) y \# 19 (Tiene una actitud amigable y de confianza con los estudiantes), que son algunas de las preguntas más importantes de la dimensión actitudinal, han ocupado los primeros lugares en todas las facultades.

Las preguntas que tienen que ver con las competencias científico-académicas de los docentes, por su parte, han ocupado todas las posiciones tanto en la Modalidad Presencial como Semipresencial: desde los primeros lugares hasta los últimos, pasando por posiciones intermedias. Es interesante, sin embargo, destacar que las preguntas \# 1 (El docente/tutor demuestra estar suficientemente preparado para el desempeño de la materia) y \# 18 (El nivel de exigencia del profesor/tutor es alto) han ocupado los primeros lugares y posiciones altas en la mayor parte de facultades a lo largo de los tres últimos semestres.

Por último, tenemos que las preguntas que aluden a las competencias metodológicas de los docentes se han ubicado, fundamentalmente, en posiciones intermedias, bajas y los últimos lugares. Vale aclarar, sin embargo, que el hecho de que estas preguntas hayan ocupado posiciones bajas no significa que tengan un promedio bajo (todas las dimensiones del desempeño docente han alcanzado un promedio alto), sino que se encuentran en un peldaño inferior a las preguntas referidas a la dimensión actitudinal y de las competencias científico-académicas.

Ahora bien, ¿qué implica que las preguntas que se refieren a la dimensión metodológica se ubiquen en posiciones bajas y en los últimos lugares? A primera vista, este dato evidenciaría un déficit pedagógico por parte de los docentes. Sin embargo, si analizamos una por una las preguntas de dicha dimensión, la interpretación cambia de perspectiva. Tomemos como ejemplo las dos preguntas 
que han ocupado posiciones bajas y los últimos lugares en todas las facultades a lo largo de estos tres últimos semestres.

La pregunta \# 4 (Es comprensible, claro y explicativo en sus clases) ha ocupado posiciones bajas y los últimos lugares en todas las facultades. Desde un ejercicio interpretativo espontáneo se podría argumentar que el dato anterior evidencia, definitivamente, un déficit en las competencias pedagógicas de los docentes: los docentes no son totalmente comprensibles, claros y explicativos en sus clases debido a que desconocen y carecen las herramientas pedagógicas - didácticas necesarias para enseñar los contenidos de su materia.

Sin desconocer que puedan haber casos de este tipo, pensamos que dicha situación puede deberse también a otro factor, a saber, al hecho de que los docentes no manejan fluidamente el campo (científico o tecnológico) que les ha sido encargado desarrollar en clase. En efecto, un docente que no maneja fluidamente su campo no está en condiciones de explicarlo por más que ponga en juego los últimos desarrollos de la pedagogía, la didáctica e inclusive, la comunicación.

Vale aclarar que cuando hablamos de "manejo fluido del campo" en ningún momento hacemos alusión a la capacidad profesional de los docentes sino al nivel de comprensión que estos tienen de sus respectivos campos: un administrador de empresas puede ser un excelente profesional pero puede ser que no conozca suficientemente su campo como para convertirlo en objeto de docencia.

Otro factor que posiblemente explica la anterior situación tiene que ver con las expectativas de los estudiantes en torno al ejercicio docente. Ciertamente, los estudiantes muchas veces esperan que sus docentes, al igual que en el colegio, les transmitan conocimientos sobre diferentes áreas de la realidad o de la ciencia. Desde esta perspectiva, es factible que los docentes que priorizan métodos más acordes con la docencia universitaria por sobre la transmisión de conocimientos, sean percibidos por los estudiantes como docentes poco claros y explicativos. 
Al igual que la pregunta \# 4, la pregunta \# 8 (Periódicamente analiza y sintetiza los temas claves de la materia) ha ocupado posiciones bajas y los últimos lugares en todas las facultades. Nuevamente este dato podría ser leído como el indicador de un déficit pedagógico de los docentes: estos no analizan ni sintetizan frecuentemente los temas claves de sus materias debido a que desconocen el significado del proceso de enseñanza así como sus principales estrategias.

Es probable que el argumento anterior tenga validez para ciertos casos, pero si nos detenemos a analizar un momento el dato, la interpretación cambia. El hecho de que los estudiantes consideren que los docentes no analizan ni sintetizan frecuentemente los temas claves de sus materias puede deberse también a otros dos factores:

- Al estilo de la docencia que priorizan los docentes y/o;

- A que los estudiantes desconocen cuáles son los temas claves de las materias.

En cuanto al primer punto, es posible que la poca frecuencia con la que los docentes analizan y sintetizan los temas claves de sus materias se deba a que están más preocupados -y en ciertos casos, hasta presionados- en transmitir abundante cantidad de conocimientos a los estudiantes que en formar su pensamiento (especificidad de la docencia universitaria). Esto es muy factible si se tiene en cuenta la cultura educativa que predomina en las instituciones universitarias del país: no nos olvidemos que para el sentido común, el mejor docente es aquel que más conocimientos logra transmitir a los estudiantes en un período de tiempo determinado.

Respecto al segundo punto, es factible que los estudiantes desconozcan cuáles son los temas claves de las materias que reciben debido a que desconocen el objeto de estudio de aquellas, sus objetivos, los contenidos y su secuencia: hasta el momento, en ninguna facultad, los docentes entregan los planes analíticos a los estudiantes. Cierto es que los profesores al inicio de cada semestre presentan a los estudiantes la materia que tienen a su cargo, pero también es cierto que solo con el plan analítico en sus manos y 
con una explicación detallada sobre su utilidad, los estudiantes están en condiciones de hacer un seguimiento de ella y de conocer cuáles son sus temas claves.

En conclusión, el hecho de que los estudiantes hayan asignado a las preguntas de la dimensión de las competencias metodológicas posiciones bajas y los últimos lugares, no solo expresa un déficit pedagógico por parte de los docentes, sino que evidencia otras situaciones que rebasan lo estrictamente pedagógico: el nivel de preparación científico-académica de los docentes, el estilo de docencia que predomina en la universidad, las expectativas de los estudiantes en torno a la docencia, el nivel de información que los estudiantes tienen de la estructura académica de las carreras, así como de cada una de las materias, entre otros.

\section{LAS DEBILIDADES CIENTÍFICO - ACADÉMICAS DEL DE- SEMPEÑO DOCENTE DESDE EL PUNTO DE VISTA DE LOS ESTUDIANTES}

Como vimos en párrafos anteriores, las preguntas de la dimensión científico-académica del desempeño docente se han ubicado, a lo largo de los tres últimos semestres, en diferentes posiciones: altas, medias y bajas. Llama la atención, sin embargo, que una de ellas haya ocupado durante los tres semestres consecutivos posiciones bajas y los últimos lugares en la mayor parte de facultades. Nos referimos a la pregunta \# 2 (Los contenidos tratados en la materia satisfacen mis expectativas).

El hecho que esta pregunta haya ocupado posiciones bajas y los últimos lugares es relevante, puesto que evidencia un desfase en el nivel de satisfacción de los estudiantes en torno a los "programas académicos" que oferta la universidad: los programas académicos se concretan, en última instancia, en los contenidos de cada una de las materias.

¿A qué se debe que los contenidos de las materias no hayan satisfecho del todo las expectativas de los estudiantes? En el nivel universitario, usualmente los contenidos de las materias no llegan a satisfacer las expectativas de los estudiantes cuando estos perciben que: 
- Los contenidos están desactualizados, esto es, que no han incorporado los últimos desarrollos de la ciencia y/o la tecnología;

- Los contenidos no son útiles para su actual o futuro ejercicio profesional;

- Los contenidos son de baja calidad, aunque estén actualizados o enfocados a su actual o futuro ejercicio.

En la UPS-Q no se ha investigado aún las causas por las que los contenidos de las materias no satisfacen del todo las expectativas de los estudiantes, pero a partir de conversaciones mantenidas con algunos grupos de ellos, se puede sostener la hipótesis de que un factor determinante de dicha situación constituye el desconocimiento que tienen de la finalidad y estructura de los programas académicos.

Ciertamente, no es raro oír a los estudiantes de algunas escuelas quejarse de la poca utilidad de las materias del área teórica o de formación básica para su futuro desempeño profesional. La queja, obviamente, se debe a un profundo desconocimiento de la finalidad del área de formación básica, que en ningún momento es proveer conocimientos y habilidades para el futuro ejercicio profesional, sino sentar las bases teóricas necesarias para la fase de especialización profesional.

Sea cual fuere la causa por la que los contenidos de las materias que se trabajan en la UPS-Q no llegan a satisfacer las expectativas de los estudiantes, es interesante notar que los posibles factores que están en su base, hacen alusión no solo a las competencias de los docentes sino también a aspectos que rebasan dichas competencias: el nivel de consistencia y pertinencia de los programas académicos que oferta la universidad, el nivel de información que los estudiantes tienen de aquellos, entre otros.

\section{CONCLUSIONES Y RECOMENDACIONES}

De las reflexiones anteriores se desprenden dos ideas importantes sobre el desempeño docente y su evaluación: 


\section{A. El desempeño docente se encuentra condicionado por fac- tores que desbordan las competencias de los docentes}

Es un lugar común pensar que el nivel de desempeño docente depende exclusivamente del nivel de las competencias que poseen los docentes. Tanto es así que se tiende a correlacionar mecánicamente dichos aspectos: a mayor nivel de competencias mayor nivel de desempeño y viceversa, así como a diseñar estrategias de mejoramiento del desempeño docente que focalizan la atención, únicamente en las competencias (por lo general pedagógicas) de los docentes.

En el presente trabajo se ha podido constatar que el desempeño docente no solo se encuentra condicionado por las competencias que los docentes poseen sino también por una serie de factores que rebasan dichas competencias: el estilo de docencia que impera en la universidad, el nivel de consistencia y pertinencia de los programas académicos, las demandas de los estudiantes en torno a la docencia universitaria, etc.; factores que pueden ser más determinantes que las mismas competencias. Poco puede hacer un docente competente cuando le asignan una materia cuyos contenidos están desactualizados y son poco pertinentes. De igual manera, poco puede hacer un docente competente cuando los estudiantes esperan que aquel se limite a transmitir información y, en el peor de los casos, a divertirles en clase.

No está por demás decir que cualquier esfuerzo por mejorar el desempeño docente debe apuntar tanto al fortalecimiento de las diferentes competencias de los docentes como a la redefinición de los aspectos arriba aludidos.

\section{B. Los resultados de las evaluaciones del desempeño docen- te dan cuenta de aspectos y dimensiones que rebasan el desempeño docente}

Tradicionalmente, los resultados de las evaluaciones del desempeño docente han sido asumidos como datos que dan cuenta exclusivamente del desempeño de los docentes: su nivel, fortalezas, debilidades, etc. 
A lo largo de este estudio, sin embargo, se ha podido evidenciar que dichos resultados, además de proveer información sobre el desempeño docente, dan cuenta sobre una serie de aspectos claves del quehacer universitario: el nivel de las diferentes competencias de los docentes, las concepciones que los diferentes actores tienen de la docencia universitaria, el nivel de consistencia y pertinencia de los programas académicos, el nivel de información que los estudiantes tienen de dichos programas, entre otros.

En esa medida, la información que proveen las evaluaciones puede ser utilizada no solo para diseñar programas de mejoramiento del desempeño docente, sino también para diseñar estrategias de desarrollo y mejoramiento curricular, programas de investigación sobre ciertas problemáticas claves del quehacer universitario (causas por las que los estudiantes de x carrera no se sienten satisfechos con los contenidos de las materias que reciben, por ejemplo), estrategias de información y comunicación de vital importancia (estrategias orientadas a informar a los estudiantes sobre la estructura académica de los programas de la universidad, por ejemplo), entre otros.

De las reflexiones anteriores se derivan también algunas recomendaciones para cualificar el proceso de evaluación del desempeño docente:

a. En primer lugar, es conveniente realizar una evaluación detallada de los indicadores e instrumentos de evaluación utilizados hasta el momento. Una evaluación de este tipo permitirá no solo conocer a profundidad el estado de dichos aspectos, sino también implantar cambios orientados a pulirlos y mejorarlos. No nos olvidemos que la validez de los resultados de las evaluaciones depende, entre otros factores, del nivel de precisión de los indicadores y de los instrumentos de evaluación.

b. En segundo lugar, es necesario impulsar un proceso de unificación y cualificación de los criterios con los que los estudiantes evalúan el desempeño de sus docentes. Decimos cualificación y no solamente unificación, debido a que la calidad de los criterios de evaluación es tan importante como el hecho de que los estudiantes manejen criterios similares. 
Dicho proceso podría ser llevado adelante por medio de un programa sostenido de información sobre las características de la docencia universitaria. Solo cuando los estudiantes comprendan cuál es la finalidad, naturaleza y método de la docencia universitaria, estarán en condiciones de evaluar el desempeño docente a partir de criterios afines a los principios de la docencia universitaria.

c. Por último, es necesario ampliar el proceso de evaluación hacia otras dimensiones. Sin desconocer que el desempeño docente es una de las dimensiones más importantes del componente docencia, pensamos que es necesario evaluar sus otras dos dimensiones: la estabilidad académica de los docentes y su formación profesional. Ambos aspectos no solo condicionan el nivel de desempeño docente sino también la calidad de los programas académicos que oferta la universidad. 